\title{
El Papel de la Personalidad y la Inteligencia en la Autoeficacia Interpersonal
}

\author{
Personality and Intelligence: Do They Affect the Interpersonal Self-Efficiency?
}

\author{
Adelia de Miguel $^{1}$, Rosario J. Marrero ${ }^{1}$, Ascensión Fumero ${ }^{1}$ y Mónica Carballeira ${ }^{1}$
}

\section{Resumen}

Se analizan las características de temperamento, inteligencia y trastornos de personalidad de 320 mujeres y 114 hombres universitarios según su autoeficacia interpersonal. Los participantes cumplimentaron el inventario de personalidad NEO-PI-R, el cuestionario de trastornos de personalidad IPDE, el cuestionario de autoevaluación de inteligencias múltiples CAIM, y los tests de factor g y de aptitudes mentales primarias PMA. Las mujeres con alta autoeficacia interpersonal puntuaron más alto en capacidad espacial, extraversión, apertura y responsabilidad, y más bajo en neuroticismo y trastornos de personalidad que las mujeres con baja autoeficacia. Los hombres del grupo alto puntuaron más alto en temperamento y más bajo en los trastornos paranoide y dependiente que los hombres del grupo bajo. Aparecieron diferencias intersexo en inteligencia en el grupo de alta autoeficacia, y en temperamento en el grupo de baja autoeficacia. Se sugiere considerar el temperamento, la inteligencia y el sexo para evaluar y promover la inteligencia interpersonal.

Palabras clave: inteligencia interpersonal, personalidad, autoevaluación, autoeficacia, género

\begin{abstract}
This study analyses temperament, intelligence, and personality disorders shown by 320 women and 114 men undergraduates depending on interpersonal self-efficacy as high or low. All of them fulfilled the Personality Inventory NEO-PI-R, the Questionnaire of Personality Disorders IPDE, the Questionnaire of SelfAssessment of Multiple Intelligences CAIM, g-factor Test, and the Primary Mental Abilities Test. Women who assessed their interpersonal intelligence as high, scored higher in spatial ability, extraversion, openness, and conscientiousness, and lower in neuroticism and personality disorders against those women who assessed their interpersonal intelligence as low. However, those men with high interpersonal self-efficacy showed higher scores on temperament and lower scores in paranoid and dependent disorders than men with low self- efficacy. There were gender differences in intelligence for the higher group in interpersonal intelligence, and in temperament for the lower group. Considering gender and personality factors in assessment and promotion of interpersonal intelligence is suggested.
\end{abstract}

Keywords: interpersonal intelligence, personality, self-assessment, self-efficacy, gender

\footnotetext{
${ }^{1}$ Sección de Psicología, Facultad de Ciencias de la Salud, Campus de Guajara 38200 La Laguna, Tenerife, España. Correo: admiguel@ull.edu.es (correspondencia Adelia de Miguel)
} 


\section{Introducción}

La inteligencia interpersonal se ha definido como la capacidad para identificar y responder adecuadamente al estado de ánimo, temperamento, motivaciones y deseos de los otros, así como saber trabajar de forma cooperativa con ellos (Gardner, 1983). Esta concepción se ha centrado en la adaptación al contexto a través de la resolución satisfactoria de situaciones interpersonales, en la que destaca la competencia de los individuos (Barnes \& Sternberg, 1989). Una persona con alta inteligencia interpersonal no sólo se comporta socialmente de una manera determinada sino que, a nivel cognitivo, representa eficientemente a otras personas y situaciones sociales y es capaz de razonar con este conocimiento (Castelló \& Cano, 2011).

Existe influencia recíproca entre las relaciones interpersonales y las características de personalidad (Asendorpf \& Wilpers, 1998) al menos hasta la edad de 30 años en la que la personalidad parece estabilizarse (McCrae \& Costa, 1990). De hecho, los rasgos de cordialidad y responsabilidad, que reflejan una cierta regulación emocional en contextos interpersonales y laborales (Larsen, 2000), aparecen asociados al número de relaciones interpersonales y a la resolución de conflictos con los otros (Asendorpf \& Wilpers, 1998). Una mayor apertura a la experiencia y un menor neuroticismo están asociados a una mayor capacidad de resolución de problemas interpersonales (Chamorro-Premuzic, Moutafi, \& Furnham, 2005). Finalmente, la satisfacción global con las relaciones interpersonales está vinculada con alta extraversión y bajo neuroticismo, y con la capacidad para manejar las propias emociones (Lopes, Salovey, \& Straus, 2003).

Además, los trastornos de personalidad, entendidos como patrones inflexibles y desadaptativos de rasgos (APA, 2013), se han relacionado con una alteración de la respuesta empática en las interacciones sociales y una capacidad reducida para llegar a soluciones efectivas ante los problemas interpersonales (Thoma, Friedmann, \& Suchan, 2013). Aunque han sido los trastornos de personalidad de tipo emocional (cluster B) los más estudiados (Vyskocilova \& Prasko, 2013), la evidencia indica que las características clínicas de cada trastorno de la personalidad se asocian a las actuaciones en tareas sociales específicas (Leising, Krause, Köhler, Hinsen, \& Clifton, 2011). Por ejemplo, los individuos con trastorno de la personalidad límite suelen presentar un estilo de solución de problemas impulsivo (McMurran, 2007) y presentan dificultades en la gestión y comprensión emocional (Gardner \& Qualter, 2009).

Por otra parte, la inteligencia auto-percibida parece estar conectada con medidas objetivas de capacidad cognitiva o de rendimiento académico (Ackerman \& Wolman, 2007; ChamorroPremuzic, Harlaar, Greven, \& Plomin, 2010). La autoevaluación de la inteligencia ha mostrado ser útil no sólo por la mayor rapidez de administración de la prueba sino porque facilita la accesibilidad a mayor población (Paulus, Lysy, \& Yik, 1998). Sin embargo, otros estudios informan de correlaciones inferiores a .30 entre inteligencia objetiva y auto-evaluada (Freund \& Kasten, 2012; Rammstedt \& Rammsayer, 2002).

Así, sentirse competente tendría que ver en parte con el rendimiento previo, pero también con la creencia de autoeficacia (Chamorro-Premuzic et al., 2010). Las creencias sobre las propias capacidades pueden tener consecuencias conductuales y motivacionales (Dweck \& Molden, 2005), reflejando incluso diferencias de tipo social, cultural y de género (Furnham, LiPing, Lester, O'Connor, \& Montgomery, 2002).

Además, se ha sugerido que las personas inteligentes tienden a mostrar mayor sensibilidad interpersonal, disponiendo de ciertas habilidades, específicamente en la decodificación de señales no verbales y en la evaluación del ámbito social (Murphy \& Hall, 2011). Leising et al. (2011) encontraron asociaciones significativas entre la imagen que tienen los individuos de sí mismos a nivel interpersonal y su evaluación del rendimiento en tareas de interacción social.

Teniendo en cuenta la capacidad dinámica de la inteligencia interpersonal y que gran parte de la misma se refleja en las interacciones del individuo con los otros, consideramos que hay una cierta metacognición que permite a la persona evaluar la eficacia de sus interacciones sociales. Sin embargo, se desconoce si son factores $\mathrm{N}^{\circ} 44 \cdot \operatorname{Vol} .2 \cdot 16-27 \cdot 2017$ 
temperamentales o intelectuales los que están implicados en la autoeficacia interpersonal.

Por lo tanto, el objetivo de este trabajo es analizar las diferencias en inteligencia y en dimensiones de personalidad (tendencias básicas y sus trastornos) entre individuos con alta o baja autoeficacia interpersonal. Además, distintos estudios han encontrado diferencias entre hombres y mujeres, tanto en capacidades intelectuales como en la autoevaluación de la inteligencia (Hyde, 2014; Thurstone \& Thurstone, 1976), por lo que un segundo propósito es analizar si existe un perfil temperamental-intelectual diferencial entre ambos sexos que incida en la autoeficacia interpersonal.

\section{Método}

\section{Muestra}

En este estudio participaron 320 mujeres $(M=20.86, D T=3.17)$ y 114 hombres $(M=21.98$, $D T=4.28)$ estudiantes de Grado de la Universidad de La Laguna. Se encontraron diferencias significativas en edad, siendo los hombres mayores que las mujeres ( $\mathrm{t}=2.93, p=.004)$.

\section{Instrumentos}

Test de Aptitudes Mentales Primarias PMA (Thurstone \& Thurstone, 1976). Consta de cinco subtests que evalúan de forma independiente la comprensión verbal, el razonamiento, la capacidad numérica, la capacidad espacial y la fluidez verbal. La prueba es administrada por un evaluador que controla el tiempo preestablecido para cada subtest (de 4 a 6 minutos). La cumplimentación total supone unos 60 minutos incluyendo instrucciones y ejercicios preparatorios. La consistencia interna del test en su versión original ha oscilado desde .73 para fluidez verbal hasta .99 para capacidad numérica para estudiantes universitarios. En este trabajo se utilizan las puntuaciones centiles de los cinco factores obtenidas a partir del baremo adaptado a la población española.

Factor g (Cattell \& Cattell, 1973). Prueba que mide el Factor General de inteligencia libre de influencias socioculturales. Está organizado en cuatro tareas (matrices, series, clasificación y condiciones) que permiten obtener una puntuación global equivalente al cociente intelectual. La aplicación requiere de un examinador que explique y controle el tiempo estipulado por la prueba, y la cumplimentación de la misma supone alrededor de 30 minutos para las instrucciones y el trabajo efectivo. La consistencia interna del test en su versión original ha sido de .75 . En este trabajo se utiliza la puntuación centil, una vez transformada la puntuación directa de acuerdo al baremo de la prueba para población española publicado por TEA Ediciones en 1984.

Cuestionario de autoevaluación de las inteligencias múltiples CAIM (Stecconi, 2006). Es una medida diseñada para evaluar la autoeficacia en las siete inteligencias múltiples propuestas por Gardner (1983) en su primera versión del modelo. Para cada una de ellas se usan 10 elementos con respuesta en escala tipo Likert de once puntos ( $0=$ totalmente en desacuerdo, $10=$ totalmente de acuerdo). En este trabajo solo se utilizará la escala de inteligencia interpersonal cuya puntuación máxima es 100 y que ha mostrado una consistencia interna adecuada de .79. Ejemplos de los ítems son: "al observar las conductas de otras personas, me doy cuenta de las emociones que sienten"; "obtengo los resultados deseados como líder o conductor de grupos"; "pido lo que necesito de forma amable, directa y veraz".

Inventario de personalidad NEO-PI-R (Costa $\&$ McCrae, 1992). Consta de 240 ítems que miden las cinco tendencias básicas del modelo de los Cinco Grandes (McCrae et al., 2000). A cada ítem se responde con una escala de intervalo tipo Likert de 5 puntos (desde $0=$ total desacuerdo hasta 4=totalmente de acuerdo). La puntuación máxima para cada gran factor es 192. Los valores del $\alpha$ de Cronbach son .89 para neuroticismo y extraversión, .85 para apertura a la experiencia y cordialidad, y .93 para responsabilidad.

Examen internacional de los trastornos de personalidad IPDE (Loranger, 1997). Es un instrumento elaborado expresamente para medir los trastornos de personalidad recogidos por la Clasificación Internacional de Enfermedades. Los 59 ítems que forman el cuestionario rastrean la posible existencia de los nueve trastornos de la personalidad. En este trabajo, se consideran como dimensiones del constructo y no como categorías diagnósticas. La respuesta a los ítems es dicotómica (SI/NO) en función de la presencia de 
esa conducta en los últimos cinco años. La consistencia interna de la prueba varía desde .73 para el trastorno anancástico hasta .88 para los trastornos disocial y dependiente.

\section{Procedimiento}

La muestra estuvo formada por estudiantes voluntarios de la Universidad de La Laguna. A lo largo de una semana, los participantes cumplimentaron las dos pruebas de inteligencia en el aula desconociendo lo que medía cada prueba. $\mathrm{Y}$ en su domicilio cumplimentaron las otras tres medidas de autoinforme sobre inteligencias múltiples, tendencias básicas y trastornos de personalidad. Posteriormente, se les informó del objetivo de la investigación, así como que se mantendría la confidencialidad y anonimato acerca de los datos recabados y se les solicitó el consentimiento informado para emplear esta información con fines de investigación.

\section{Análisis estadístico}

Para el análisis de los datos se empleó el programa IBM SPSS (versión 19.0). Las diferencias entre grupos se calcularon con la $t$ de Student y la $d$ de Cohen. Se analizaron las diferencias entre ambos sexos en autoeficacia interpersonal. El análisis de frecuencias permitió establecer dos grupos extremos en autoeficacia interpersonal (bajo y alto). Se analizaron las diferencias entre dichos grupos en inteligencia, tendencias básicas y trastornos de personalidad, primero sin tener en cuenta el sexo de los participantes y después considerando las diferencias intrasexo entre ellos. Posteriormente se analizaron las diferencias intersexo dentro de cada grupo extremo en autoeficacia interpersonal. Y por último, se llevaron a cabo tres análisis discriminantes (con toda la muestra y para cada sexo) para identificar las características intelectuales y temperamentales que definían a los participantes de los grupos alto y bajo en autoeficacia interpersonal.

\section{Resultados}

En general, los participantes mostraron una alta autoeficacia interpersonal (media $\geq 70$ ), $\sin$ diferencias intersexo $(t=1.69, p=.93)$.
A partir de la puntuación obtenida en la escala de autoeficacia interpersonal, se establecieron dos grupos extremos: el grupo bajo correspondía al primer cuartil $(N=97$, media $=60.12, \sigma=7.61)$ y el grupo alto al cuarto cuartil $(N=115$, media $=84.54$, $\sigma=3.80$ ). La composición de ambos grupos no mostró diferencias en la distribución por sexos $\left(\chi^{2}=1.40, g l=1, p=.24\right)$ y eran homogéneos en edad $(t=-.88, p=.38)$.

En la Tabla 1 se recogen las medias y las desviaciones típicas de todas las variables para toda la muestra en función del sexo y para los grupos extremos en autoeficacia interpersonal, tanto en general como por sexo.

\section{Diferencias en inteligencia, tendencias básicas y trastornos de personalidad entre los grupos extremos en autoeficacia interpersonal}

En primer lugar, independientemente del sexo, los resultados indicaron que las personas del grupo bajo en autoeficacia interpersonal frente a las del grupo alto rendían mejor en el factor espacial, se mostraban más inestables emocionalmente, eran más introvertidas, menos abiertas a la experiencia, menos cordiales, menos responsables en las tareas laboral/académicas, y puntuaban más alto en todos los trastornos de personalidad - excepto el histriónico y el anancástico (véase Tabla 2).

Este patrón diferencial se repitió cuando se comparó a las mujeres del grupo bajo con las mujeres del grupo alto, aunque desaparecieron las diferencias en el rasgo de cordialidad y en el rasgo de personalidad disocial, y surgió una diferencia en el rasgo histriónico, puntuando más alto las mujeres del grupo bajo en autoeficacia interpersonal.

En el caso de los hombres, los resultados indicaron un patrón semejante al de la muestra total, similitud en los factores de inteligencia (aunque desapareció la diferencia en el factor espacial) entre ambos grupos extremos; menores puntuaciones en estabilidad emocional, extraversión, apertura a la experiencia, cordialidad y responsabilidad para el grupo bajo; y solo diferencias en los rasgos de personalidad paranoide y dependiente, puntuando más alto en ambos los hombres del grupo bajo en autoeficacia interpersonal. 
Tabla 1. Medias y desviaciones típicas en todos los factores evaluados

Toda la muestra Grupo bajo en Grupo alto en autoeficacia Grupo total Grupo total autoeficacia interpersonal interpersonal bajo en alto en

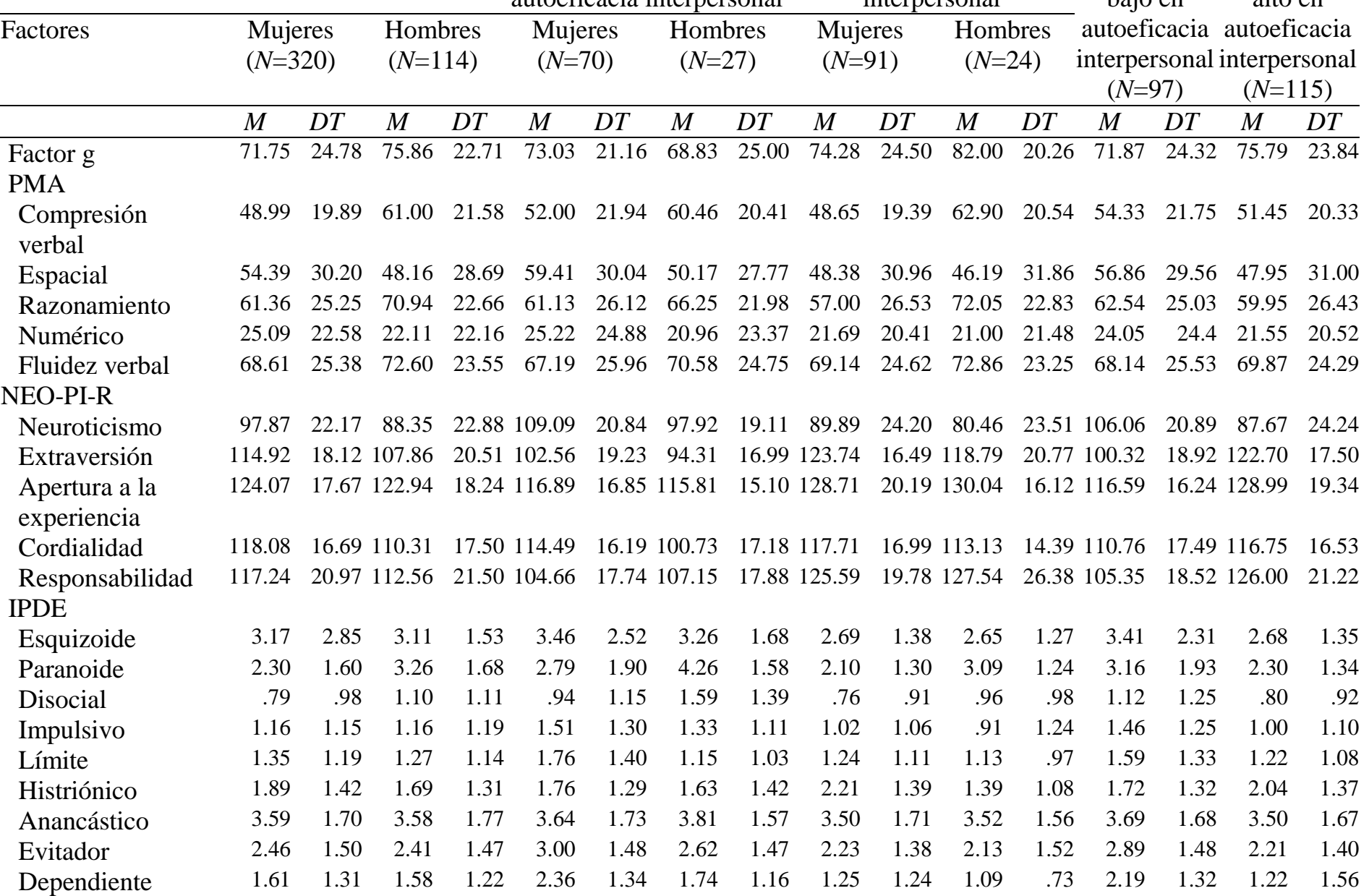

PMA: Test de Aptitudes Mentales Primarias PMA; NEO-PI-R: Inventario de personalidad NEO-PI-R; IPDE:

Examen internacional de los trastornos de personalidad IPDE

\section{Diferencias de sexo en inteligencia, tendencias} básicas y trastornos de personalidad en función de la autoeficacia interpersonal

Independientemente de la autoeficacia interpersonal, mujeres y hombres no se diferenciaron en el factor $g$ ni en los factores espacial, numérico y fluidez verbal; pero sí en los factores de comprensión verbal y razonamiento, en los que puntuaron más alto los hombres. En el área temperamental, las mujeres puntuaron más alto que los hombres en los rasgos de neuroticismo, extraversión, cordialidad y responsabilidad. En cuanto a los trastornos de personalidad solo hubo dos diferencias significativas, los hombres puntuaron más alto que las mujeres en los rasgos paranoide y disocial (véase Tabla 3).

Dentro del grupo bajo en autoeficacia interpersonal, mujeres y hombres puntuaron de forma similar en todos los factores de inteligencia; pero las mujeres se mostraron más inestables emocionalmente y más cordiales que los hombres, tuvieron una puntuación más alta en los rasgos límite y dependiente, y una puntuación más baja en los rasgos paranoide y disocial.

Finalmente, en el grupo alto en autoeficacia interpersonal, no hubo diferencias entre ambos sexos ni en temperamento ni en el factor $g$. Sin embargo, los hombres puntuaron más alto que las mujeres en los factores de comprensión verbal y razonamiento, y en el rasgo paranoide; y más bajo en el rasgo histriónico.

\section{Perfil de mujeres y hombres en función de la autoeficacia interpersonal}

Se aplicó un análisis discriminante paso a paso para identificar si existían capacidades intelectuales, tendencias básicas de personalidad y sus trastornos que caracterizasen a los individuos con baja o alta autoeficacia interpersonal. En la Tabla 4 se incluyeron únicamente los coeficientes 
Tabla 2. Diferencias entre grupos extremos de autoeficacia interpersonal en variables intelectuales, tendencias básicas y trastornos de personalidad, para la muestra total y en función del sexo

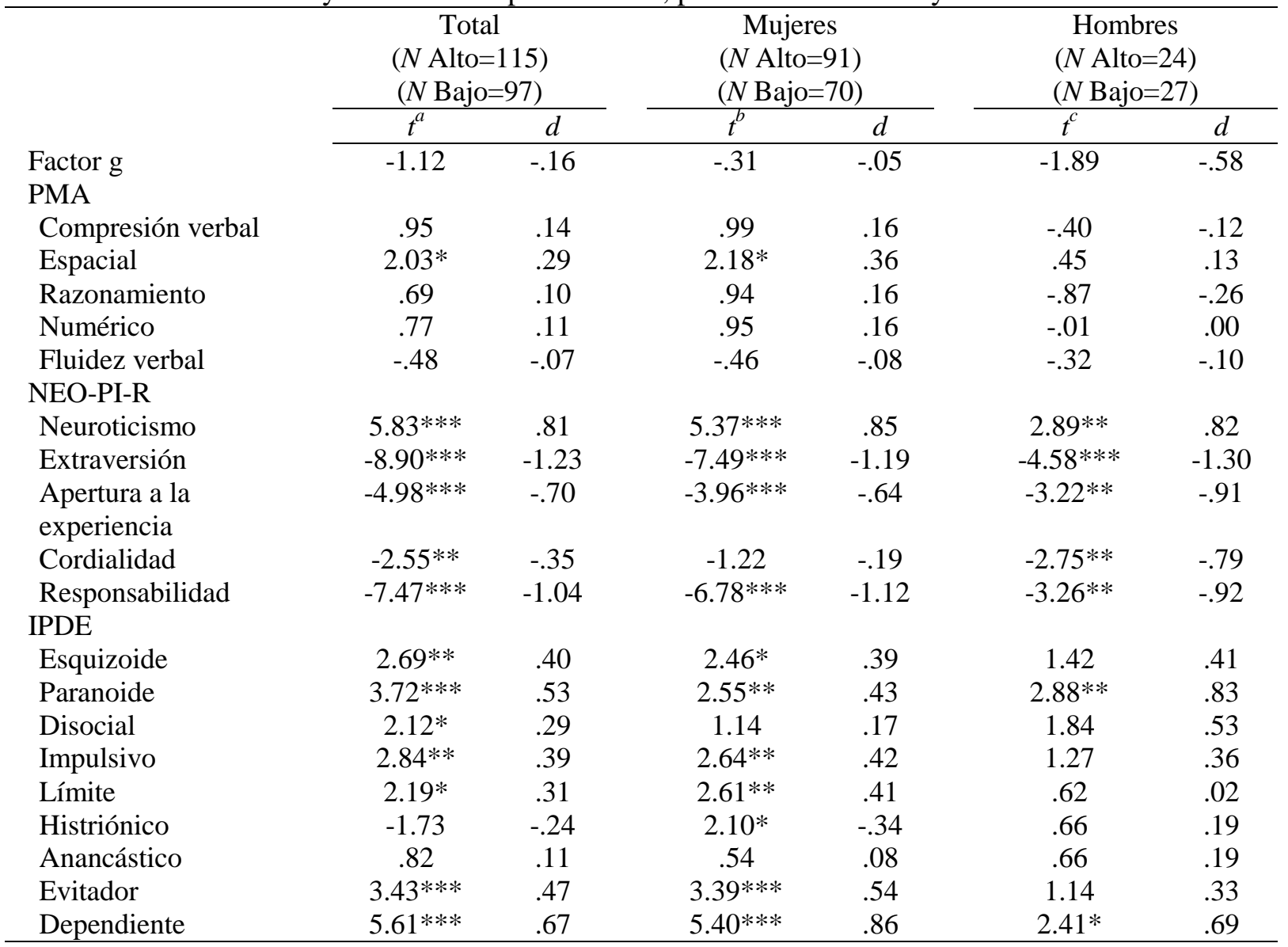

PMA: Test de Aptitudes Mentales Primarias PMA; NEO-PI-R: Inventario de personalidad NEO-PI-R; IPDE: Examen internacional de los trastornos de personalidad IPDE

${ }^{\mathrm{a}} g l=210 ;{ }^{\mathrm{b}} \mathrm{gl}=159 ;{ }^{\mathrm{c}} \mathrm{gl}=49$

$t=t$ de Student; $d=d$ de Cohen; * $p<.05 ; * * p<.01 ; * * * p<.001$

tipificados de las variables que formaron la función discriminante.

Cuando se analizó la muestra conjuntamente, la función discriminante permitió identificar a las personas del grupo alto frente a las del grupo bajo en autoeficacia interpersonal como más extravertidas, responsables, buscadoras de sensaciones, con mayor puntuación en el rasgo límite y menor en el dependiente. Esta función permitió clasificar correctamente al $82.8 \%$ de los participantes $(85.4 \%$ para el grupo bajo y $80.5 \%$ para el grupo alto).

En el análisis llevado a cabo para el grupo de mujeres de alta autoeficacia interpersonal, se obtuvo que eran extravertidas, responsables y tenían menor puntuación en el rasgo de personalidad dependiente pero mayor en el evitativo que las mujeres del grupo bajo. La función clasificó correctamente al $81.9 \%$ de los casos, siendo los porcentajes similares para los grupos bajo y alto en autoeficacia interpersonal.

Finalmente, en el caso de los hombres, la función discriminante incluyó dos rasgos temperamentales: los hombres de alta autoeficacia interpersonal eran más extravertidos y responsables que los del grupo bajo. Los porcentajes de clasificaciones correctas fueron $92.3 \%$ para el grupo bajo y $79.2 \%$ para el grupo alto.

En los tres análisis discriminantes efectuados ningún factor de inteligencia objetiva entró a formar parte de la función discriminante.

Considerando la autoeficacia interpersonal como variable continua, se realizaron también tres regresiones lineales (toda la muestra, mujeres y hombres). Los valores de las $R^{2}$ recogidos en la Tabla 5 variaron entre .30 y $.33(p<.001)$. Los coeficientes tipificados $\beta$ proporcionaron información adicional, ya que para el grupo de los 
Tabla 3. Diferencias entre hombres y mujeres en variables intelectuales, tendencias básicas y trastornos en función del nivel de autoeficacia interpersonal

\begin{tabular}{|c|c|c|c|c|c|c|}
\hline & \multicolumn{2}{|c|}{$\begin{array}{c}\text { Total } \\
(N \text { Mujeres }=320) \\
(N \text { Hombres }=114)\end{array}$} & \multicolumn{2}{|c|}{$\begin{array}{c}\text { Grupo Bajo } \\
(N \text { Mujeres }=70) \\
(N \text { Hombres }=27)\end{array}$} & \multicolumn{2}{|c|}{$\begin{array}{c}\text { Grupo Alto } \\
(N \text { Mujeres }=91) \\
(\mathrm{N} \text { Hombres }=24)\end{array}$} \\
\hline & $t^{a}$ & $d$ & $t^{b}$ & $d$ & $t^{c}$ & $d$ \\
\hline Factor $\mathrm{g}$ & -1.44 & -.17 & 0.72 & .18 & -1.30 & -.34 \\
\hline \multicolumn{7}{|l|}{ PMA } \\
\hline $\begin{array}{l}\text { Compresión } \\
\text { verbal }\end{array}$ & $-5.15 * * *$ & -.58 & -1.67 & -.40 & $-2.99 * *$ & -.71 \\
\hline Espacial & 1.82 & .21 & 1.31 & .32 & .29 & .07 \\
\hline Razonamiento & $-3.39 * *$ & -.40 & -.86 & -.21 & $-2.39 *$ & -.61 \\
\hline Numérico & 1.15 & .13 & .73 & .18 & .14 & .03 \\
\hline Fluidez verbal & -1.39 & -.16 & -.55 & -.13 & -.63 & -.16 \\
\hline \multicolumn{7}{|l|}{ NEO-PI-R } \\
\hline Neuroticismo & $3.88^{* * *}$ & .42 & $2.38 *$ & .56 & 1.65 & .40 \\
\hline Extraversión & $3.43^{* * *}$ & .37 & 1.93 & .46 & 1.24 & .27 \\
\hline $\begin{array}{l}\text { Apertura a la } \\
\text { experiencia }\end{array}$ & .58 & .06 & .29 & .07 & -.30 & -.07 \\
\hline Cordialidad & $4.17 * * *$ & .45 & $3.64 * * *$ & .82 & 1.21 & .29 \\
\hline Responsabilidad & $2.03^{*}$ & .22 & -.59 & -.14 & -.40 & -.08 \\
\hline \multicolumn{7}{|l|}{ IPDE } \\
\hline Esquizoide & .23 & .03 & .38 & .10 & .13 & .03 \\
\hline Paranoide & $-5.38 * * *$ & -.59 & $-3.68 * * *$ & -.84 & $-3.29 * * *$ & -.78 \\
\hline Disocial & $-2.78 * *$ & -.30 & $-2.34 *$ & -.51 & -.92 & -.21 \\
\hline Impulsivo & .01 & .00 & .64 & .15 & .42 & .10 \\
\hline Límite & .56 & .07 & $2.06^{*}$ & .50 & .44 & .11 \\
\hline Histriónico & 1.30 & .15 & .42 & .10 & $2.62 * *$ & .66 \\
\hline Anancástico & .10 & .01 & -.45 & -.10 & -.07 & -.01 \\
\hline Evitador & .30 & .03 & 1.13 & .26 & .31 & .07 \\
\hline Dependiente & .24 & .02 & $2.10 *$ & .50 & .61 & .16 \\
\hline
\end{tabular}

PMA: Test de Aptitudes Mentales Primarias PMA; NEO-PI-R: Inventario de personalidad NEO-PIR; IPDE: Examen internacional de los trastornos de personalidad IPDE

${ }^{\mathrm{a}} g l=432 ;{ }^{\mathrm{b}} g l=95 ;{ }^{\mathrm{c}} g l=113$

$t=t$ de Student; $d=d$ de Cohen; ${ }^{*} p<.05 ; * * p<.01 ; * * * p<.001$

hombres, obtener una puntuación baja en los trastornos límite e impulsivo predecía una mayor puntuación en autoeficacia interpersonal. El resto de valores se solapaban con los obtenidos en los análisis discriminantes.

\section{Discusión y conclusiones}

El objetivo de este trabajo fue identificar si las características temperamentales e intelectuales de los participantes incidían en la autoeficacia interpersonal, al tiempo que se analizaban las posibles diferencias entre hombres y mujeres. En general, los resultados indican que la alta autoeficacia interpersonal está caracterizada por un perfil temperamental-intelectual específico, con variaciones en función del sexo.

En el presente trabajo se consideró la autoevaluación como una estrategia útil para detectar la inteligencia interpersonal. Esta técnica responde de una manera rápida, accesible, con poca inversión personal y, por lo tanto, de forma más parsimoniosa a la evaluación de un constructo. Además, resultaba necesario identificar si las características temperamentales e intelectuales estaban implicadas en la autoeficacia interpersonal.

Se trabajó con tres áreas de la personalidad con relaciones teóricas y actuariales con la inteligencia interpersonal. Esas áreas fueron la inteligencia (general y aptitudes primarias), las tendencias básicas y los trastornos de la personalidad (desde una perspectiva dimensional).

El hecho de que fuese un grupo homogéneo, en el que todos los participantes se situaban por encima de la media en autoeficacia interpersonal, podría haber eliminado otras diferencias intergrupales. Sin embargo, sin tener en cuenta el 
Tabla 4. Discriminantes entre grupos extremos en autoeficacia interpersonal para la muestra total y en función del sexo

\begin{tabular}{|c|c|c|c|}
\hline & $\begin{array}{c}\text { Total } \\
(N=212) \\
\end{array}$ & $\begin{array}{l}\text { Mujeres } \\
(N=161)\end{array}$ & $\begin{array}{c}\text { Hombres } \\
(N=51)\end{array}$ \\
\hline $\begin{array}{l}\text { Correlación } \\
\text { canónica }\end{array}$ & .68 & .65 & .72 \\
\hline $\begin{array}{l}\text { Lambda de Wilks } \\
\text { Centroides }\end{array}$ & $.54 * * *$ & $.57 * * *$ & $.49 * * *$ \\
\hline $\begin{array}{l}\text { Grupo Bajo } \\
\text { Grupo Alto }\end{array}$ & $\begin{array}{c}-1.00 \\
.85\end{array}$ & $\begin{array}{l}-.99 \\
.75\end{array}$ & $\begin{array}{l}-.89 \\
1.12\end{array}$ \\
\hline $\begin{array}{l}\text { Coeficientes } \\
\text { tipificados }\end{array}$ & & & \\
\hline Extraversión & .64 & .73 & .87 \\
\hline $\begin{array}{l}\text { Apertura a la } \\
\text { experiencia }\end{array}$ & .23 & -- & -- \\
\hline Responsabilidad & .64 & .59 & .88 \\
\hline Rasgo Límite & .25 & -- & -- \\
\hline Rasgo Evitador & -- & .33 & -- \\
\hline $\begin{array}{l}\text { Rasgo } \\
\text { Dependiente } \\
\% \text { Clasificación } \\
\text { correcta }\end{array}$ & -.34 & -.42 & -- \\
\hline Total & 82.8 & 81.9 & 86.0 \\
\hline Grupo Bajo & 85.4 & 82.9 & 92.3 \\
\hline Grupo Alto & 80.5 & 81.1 & 79.2 \\
\hline
\end{tabular}

PMA: Test de Aptitudes Mentales Primarias PMA; NEO-PI-R: Inventario de personalidad NEO-PI-R; IPDE: Examen internacional de los trastornos de personalidad IPDE

$* p<.05 ; * * p<.01 ; * * * p<.001$

sexo de los participantes, ambos grupos fueron similares en inteligencia objetivamente medida (excepto en el factor espacial), pero se diferenciaron en todos los factores temperamentales y en la mayor parte de los trastornos de personalidad. Concretamente, el grupo bajo en autoeficacia interpersonal, frente al grupo alto, rindió mejor en la tarea espacial pero se mostró más inestable emocionalmente, más introvertido, menos abierto a la experiencia, menos cordial y menos responsable en las tareas académico/laborales. Estos resultados coinciden con los informados en otros estudios (ChamorroPremuzic et al., 2005; Larsen, 2000; Lopes et al., 2003). Además, el grupo bajo también obtuvo puntuaciones claramente superiores al grupo alto en los rasgos de personalidad esquizoide, paranoide, disocial, impulsivo, límite, evitativo y dependiente. Esta mayor vulnerabilidad psicológica, claramente relacionada con las relaciones interpersonales, en quienes muestran baja autoeficacia interpersonal, señala la necesidad de que los programas preventivos y Revista Iberoamericana de Diagnóstico y Evaluación - e Avaliação Psicológica. RIDEP · N44 · Vol.2 · 16-27 · 2017 terapéuticos dirigidos a tratar los trastornos de personalidad incluyan actuaciones sobre las habilidades sociales, tal y como se sugiere desde la investigación (Mckay, Gavigan, \& Kulchycky, 2004).

No obstante, los resultados anteriores son aplicables a las mujeres pero entre los hombres de ambos grupos en autoeficacia interpersonal no aparecieron diferencias en sus puntuaciones en trastornos de personalidad. Esto apoya la necesidad de un acercamiento diferencial en las intervenciones en función del sexo y la autoeficacia interpersonal.

Las diferencias intersexo dentro del grupo bajo en autoeficacia interpersonal indicaron que las mujeres eran más ansiosas pero más cordiales que los hombres, más vulnerables a las personalidades límite y dependiente, pero menos paranoides y con menos conductas antisociales. Es decir, se consideraron poco hábiles en sus relaciones interpersonales a pesar de calificarse como más dispuestas a ayudar a los demás, a preocuparse por sus problemas, a confiar en los otros y a ser más altruistas que los hombres. Las mujeres se muestran más insatisfechas consigo mismas, más tímidas y con mayor sentimiento de tristeza, pena, angustia, vergüenza, autocompasión o desdicha (Byrne, 2000).

Teniendo en cuenta que los participantes eran universitarios, los resultados apoyan la evidencia de que las mujeres más jóvenes presentan una tendencia a mostrar conductas más complacientes ante las demandas de otras personas, mientras que los hombres jóvenes se muestran más conflictivos socialmente (Siverio y García, 2007). Quizás estos resultados podrían explicarse en base a las prácticas de crianza y socialización como justificación del mantenimiento de las diferencias $\mathrm{y}$ estereotipos de género en la sociedad contemporánea. De hecho, Fivush (1991) encontró que las madres alentaban las manifestaciones de tristeza en las niñas y corregían las muestras de ira, mientras no prestaban atención a las manifestaciones de tristeza de los niños y eran más permisivas con las de ira. Estos estereotipos de género son asumidos por los adolescentes, de modo que los chicos que muestran ira y las chicas que muestran tristeza son más aceptados por sus iguales. 
Tabla 5. Regresión lineal sobre autoeficacia interpersonal para la muestra total y

\begin{tabular}{|c|c|c|c|c|c|c|}
\hline & \multicolumn{2}{|c|}{$\begin{array}{c}\text { Total } \\
(N=434)\end{array}$} & \multicolumn{2}{|c|}{$\begin{array}{l}\text { Mujeres } \\
(N=320)\end{array}$} & \multicolumn{2}{|c|}{$\begin{array}{c}\text { Hombres } \\
(N=114)\end{array}$} \\
\hline & $R^{2}$ & $\beta$ & $R^{2}$ & $\beta$ & $R^{2}$ & $\beta$ \\
\hline & .32 & & .30 & & .33 & \\
\hline Factor g & & .02 & & -.01 & & .11 \\
\hline \multicolumn{7}{|l|}{ PMA } \\
\hline Compresión verbal & & -.03 & & -.02 & & -.08 \\
\hline Espacial & & -.08 & & -.06 & & -.08 \\
\hline Razonamiento & & -.02 & & -.04 & & .08 \\
\hline Numérico & & -.01 & & -.01 & & -.05 \\
\hline Fluidez verbal & & .01 & & -.01 & & .02 \\
\hline \multicolumn{7}{|l|}{ NEO-PI-R } \\
\hline Neuroticismo & & -.05 & & -.05 & & -.09 \\
\hline Extraversión & & $.32 * * *$ & & $.34 * * *$ & & $.49 * * *$ \\
\hline Apertura a la experiencia & & $.11 * *$ & & .10 & & .15 \\
\hline Cordialidad & & $.13 * *$ & & .10 & & .13 \\
\hline Responsabilidad & & $.31 * * *$ & & $.34 * * *$ & & $.23 *$ \\
\hline \multicolumn{7}{|l|}{ IPDE } \\
\hline Esquizoide & & .01 & & .01 & & .06 \\
\hline Paranoide & & .02 & & .02 & & .02 \\
\hline Disocial & & .03 & & .02 & & -.06 \\
\hline Impulsivo & & -.07 & & -.08 & & $-.21 *$ \\
\hline Límite & & .04 & & .01 & & .07 \\
\hline Histriónico & & -.04 & & .04 & & $-.24 *$ \\
\hline Anancástico & & -.01 & & -.03 & & -.03 \\
\hline Evitador & & $.12 *$ & & $.14 *$ & & .04 \\
\hline Dependiente & & $-.21 * * *$ & & $-.20 * * *$ & & -.14 \\
\hline
\end{tabular}

PMA: Test de Aptitudes Mentales Primarias PMA; NEO-PI-R: Inventario de personalidad NEO-PI-R; IPDE: Examen internacional de los trastornos de personalidad IPDE

$* p<.05 ; * * p<.01 ; * * * p<.001$

Por el contrario, dentro del grupo alto en autoeficacia interpersonal, los hombres rendían más que las mujeres en los factores intelectuales de comprensión verbal y razonamiento, desconfiaban más de los otros pero puntuaban menos en el rasgo histriónico.

En otras palabras, las diferencias entre ambos sexos para los que se consideran poco hábiles a nivel interpersonal se centran principalmente en aspectos temperamentales (ansiedad y relaciones con los demás), mientras que son los factores intelectuales los que diferencian a hombres y mujeres en el grupo de alta autoeficacia interpersonal.

Sin embargo, los resultados hallados a través del análisis discriminante, indicaron que el temperamento clasificaba mejor que la inteligencia a las personas que mostraban una alta autoeficacia interpersonal frente a las que informaron de baja autoeficacia. Los participantes con alta autoeficacia interpersonal mostraron ser extravertidos, responsables, abiertos a la experiencia, con ciertos indicadores de rasgo límite y escasa sintomatología dependiente. Este patrón es bastante similar para hombres y mujeres, aunque para ellas se añadió la vulnerabilidad a los rasgos evitativos y dependientes, ambos pertenecientes al núcleo ansioso. Las mujeres con alta autoeficacia interpersonal aparecieron menos dependientes pero más evitadoras que los hombres altos en autoeficacia interpersonal. Una explicación tentativa para este resultado podría apelar a la mayor importancia de los aspectos emocionales en el funcionamiento psicológico de las mujeres (Altemus, Sarvaiya, \& Neill Epperson 2014; Thompson \& Voyer, 2014).

Todos estos resultados nos llevan a afirmar tentativamente que la autoeficacia interpersonal se ve afectada en gran medida por rasgos temperamentales pero no por la inteligencia objetivamente evaluada. Ni el factor $g$ ni las aptitudes primarias aparecieron en ninguna de las 
tres funciones discriminantes. Estos resultados coinciden con los de otras investigaciones (p.ej., Roberts, Zeidner, \& Matthews, 2001).

Una limitación de este trabajo es que no se ha medido la inteligencia interpersonal con una prueba de rendimiento, por lo que se desconoce la capacidad objetiva de los participantes para hacer frente a situaciones interpersonales. Además, al tratarse de una muestra de estudiantes jóvenes, los resultados deben tomarse con cautela a la hora de establecer generalizaciones a población adulta con diferentes niveles de cualificación.

En el futuro se recomienda seguir investigando la concordancia entre medidas objetivas de la inteligencia interpersonal y medidas subjetivas de autoeficacia interpersonal. Ser capaz de estimar la propia inteligencia o la de los demás ofrece información sobre qué es la inteligencia y qué formas de conducta son consideradas inteligentes para los individuos. Solo así se podrá analizar si la autoeficacia interpersonal coincide con el rendimiento interpersonal, o bien se trata de una habilidad metacognitiva en la que están implicados factores temperamentales más que factores aptitudinales. $\mathrm{Su}$ detección permitiría realizar una evaluación más precisa y diseñar programas de intervención más ajustados a las necesidades de los individuos.

\section{Referencias}

Ackerman, P. L., \& Wolman, S. D. (2007). Determinants and validity of self-estimates of abilities and self-concept measures. Journal of Experimental Psychology: Applied, 13, 57-78. http://dx.doi.org/10.1037/1076-898X.13.2.57

Adrián, L., Moretti, L., \& Ortiz, A. (2015). Medición del Engagement Académico en Estudiantes Universitarios. Revista Iberoamericana de Diagnóstico y Evaluación - e Avaliação Psicológica, 40(2), 114-123.

Altemus, M., Sarvaiya, N., \& Neill Epperson, C. (2014). Sex differences in anxiety and depression clinical perspectives. Frontiers in Neuroendocrinology, 35, 320-330. http://dx.doi.org/10.1016/j.yfrne.2014.05.004.

American Psychiatric Association (Ed.). (2013). Diagnostic and statistical manual of mental disorders: $\quad D S M-V$. American Psychiatric Publishing.

Asendorpf, J. B., \& Wilpers, S. (1998). Personality effects on social relationships. Journal of Personality and Social Psychology, 74, 1531-1544. http://dx.doi.org/10.1037/002 2-3514.74.6.1531

Barnes, M. L., \& Sternberg, R. J. (1989). Social intelligence and decoding of nonverbal cues. Intelligence, 13, 263-287. http://dx.doi.org/10. 1016/0160-2896(89)90022-6

Bravo-González, Vaquero-Cázares, \& ValadezRamírez (2012). Las competencias profesionales percibidas por estudiantes de psicología [The professional competences perceived by psychology students]. Revista de Educación y Desarrollo, 2, 13-20. Recuperado de http://www.cucs.udg.mx/revist as/edu_desarrollo/anteriores/21/021_RED_co mpleta_verfinal.pdf\#page $=14$

Byrne, B. (2000). Relationships between anxiety, fear, self-steem, and coping strategies in adolescence. Adolescence, 35, 201-215.

Castelló, A., \& Cano, M. (2011). Inteligencia interpersonal: conceptos clave [Interpersonal intelligence: key concepts]. Revista Electrónica Interuniversitaria de Formación del Profesorado, 14, 23-25. Recuperado de file://ull.local/datos/adelia\%20de\%20miguel/ Descargas/Dialnet-InteligenciaInterpersonal4620047.pdf

Cattell, R. B., \& Cattell, A.K.S. (1973). Measuring intelligence with the culture fair test. Illinois: Institute for Personality and Ability Testing (Adaptación española, TEA Ediciones, S.A, 1984).

Chamorro-Premuzic, T., Harlaar, N., Greven, C. U., \& Plomin, R. (2010). More than just IQ: A longitudinal examination of self-perceived abilities as predictors of academic performance in a large sample of UK twins. Intelligence, 38, 385-392. http://dx.doi.org/10. 1016/j.intell.2010.05.002

Chamorro-Premuzic, T., Moutafi, J., \& Furnham, A. (2005). The relationship between personality traits, subjectively-assessed and fluid intelligence. Personality and Individual Differences, 38, 1517-1528. http://dx.doi.org/ 10.1016/j.paid.2004.09.018 
Costa, P. T., \& McCrae, R. R. (1992). Normal personality assessment in clinical practice: The NEO Personality Inventory. Psychological Assessment, 4, 5-13. http://dx.doi.org/10.1037/1040-3590.4.1.5

Dweck, C. S., \& Molden, D. C. (2005). Selftheories: Their impact on competence motivation and acquisition. En A. J. Elliot y C. S. Dweck (Eds.). Handbook of Competence and Motivation (pp. 122-140). Nueva York: The Guilford Press.

Fivush, R. (1991). Gender and emotion in motherchild conversations about the past. Journal of Narrative and Life History, 1, 325-341.

Freund, P. A., \& Kasten, N. (2012). How smart do you think you are? A meta-analysis on the validity of self-estimates of cognitive ability. Psychological Bulletin, 138, 296-321. http://dx.doi.org/10.1037/a0026556

Furnham, A., Li-Ping, T., Lester, D., O'Connor, R., \& Montgomery, R. (2002). Estimates of ten multiple intelligences. Sex and national differences in the perception of oneself and famous people. European Psychologist, 7, 245-255. http://dx.doi.org/10.1027//10169040.7.4.245

Gardner, H. (1983). Frames of mind: The theory of multiple intelligences. Nueva York: Basic Books.

Gardner, K., \& Qualter, P. (2009). Emotional intelligence and borderline personality disorder. Personality and Individual Differences, 47, 94-98. http://dx.doi.org/ 10.1016/j.paid.2009.02.004

Herrera, A, Restrepo, M. F., Uribe, A., \& López, C. (2009) Competencias académicas y profesionales del psicólogo [The psychologist academic and profesional competences]. Revista Diversitas - Perspectivas en Psicología, 5, 241-254. Recuperado de file://ull.local/datos/adelia\%20de\%20miguel/ Descargas/167-570-1-PB.pdf

Hyde, J. S. (2014). Gender similarities and differences. Annual Review of Psychology, 65, 373-398. http://dx.doi.org/10.1146/annurevpsych-010213-115057

Inzunza, I., Ortiz, L, Pérez, C., Torres, G., McColl, P., Meyer, A., Matus, A., Bastías, N., $\&$ Bustamante, C. (2015). Estructura factorial y confiabilidad del Cuestionario de
Satisfacción Académica en Estudiantes de Medicina Chilenos. Revista Iberoamericana de Diagnóstico y Evaluación - e Avaliação Psicológica, 40(2), 73-82.

Larsen, R. J. (2000). Toward a science of mood regulation. Psychological Inquiry, 11, 129141. http://dx.doi.org/10.1207/S15327965PLI 1103_01

Leising, D., Krause, S., Köhler, D., Hinsen, K., \& Clifton, A. (2011). Assessing interpersonal functioning: Views from within and without. Journal of Research in Personality, 45, 631641. http://dx.doi.org/10.1016/j.jrp.2011.08.0 11

Lopes, P. N., Salovey, P., \& Straus, R. (2003). Emotional intelligence, personality, and the perceived quality of social relationships. Personality and Individual Differences, 35, 641-658. http://dx.doi.org/10.1016/S01918869(02)00242-8

Loranger, A. W. (1997). International personality disorder examination (IPDE). En A.W. Loranger, A. Janca y N. Sartorius (eds.). Assessment and diagnosis of personality disorders. The ICD-10 international personality disorder examination (IPDE). (pp. 43-51) Cambridge: Cambridge University Press.

McCrae, R. R., \& Costa, P. T. (1990). Personality in Adulthood. Nueva York: Guilford Press.

McKay, D., Gavigan, C. A., y Kulchycky, S. (2004). Social skills and sex-role functioning in borderline personality disorder: Relationship to self-mutilating behavior. Cognitive Behaviour Therapy, 33, 27-35. http://dx.doi.org/ 10.1080/1650607031000219 9

McMurran, M. (2007). What works in substance misuse treatments for offenders? Criminal Behaviour and Mental Health, 17, 225-233. http://dx.doi.org/10.1002/cbm.662

Murphy, N. A., \& Hall, J. A. (2011). Intelligence and interpersonal sensitivity: A meta-analysis. Intelligence, 39, 54-63. http://dx.doi.org/10.10 16/j.intell.2010.10.001

Paulus, D., Lysy, D., \& Yik, M. (1998). Selfreport measures of intelligence: Are they useful as proxy IQ tests? Journal of Personality, 66, 525-554. http://dx.doi.org/10. 1111/1467-6494.00023 
Rammstedt, B., \& Rammsayer, T. H. (2002). Selfestimated intelligence. European Psychologist, 7, 275-284. http://dx.doi.org/10. 1027//1016-9040.7.4.275

Roberts, R. D., Zeidner, M., \& Matthews, G. (2001). Does emotional intelligence meet traditional standards for an intelligence?. Some new data and conclusions. Emotion, 1, 196-231. http://dx.doi.org/10.1037/15283542.1.3.196

Siverio, M. A., \& García, M.D. (2007). Autopercepción de adaptación y tristeza en la adolescencia: La influencia del género [Selfperception of adjustment and sadness in adolescence]. Anales de Psicología, 23, 4148. Recuperado de http://revistas.um.es/anales ps/article/view/23211/22491

Stecconi, C. (2006). Inteligencias múltiples y el cuestionario de autoevaluación [Multiple Intelligences and the self-assessing questioinnaire] (CAIM). Calidad de Vida, 5, 147-164.

Thoma, P., Friedmann, C., \& Suchan, B. (2013). Empathy and social problem solving in alcohol dependence, mood disorders and selected personality disorders. Neuroscience \& Biobehavioral Reviews, 37, 448-470. http://dx.doi.org/10.1016/j.neubiorev.2013.01. 024

Thompson, A. E., \& Voyer, D. (2014). Sex differences in the ability to recognise nonverbal displays of emotion: A metaanalysis. Cognition \& Emotion, 28, 1-32. http://dx.doi.org/10.1080/02699931.2013.875 889

Thurstone, L. L., \& Thurstone, Th. G. (1976). PMA: Aptitudes Mentales Primarias. [Primary Mental Aptitudes] Madrid: TEA.

Vyskocilova, J., \& Prasko, J. (2013). Social skills training in psychiatry. Activitas Nervosa Superior Rediviva, 54, 159-170. Recuperado de http://www.rediviva.sav.sk/54i4/159.pdf 